Published in final edited form as:

Int J Cardiol. 2015 May 6; 187: 325-333. doi:10.1016/j.ijcard.2015.03.352.

\title{
Role of Mitochondrial fission and fusion in cardiomyocyte contractility
}

\author{
S Givvimani, SB Pushpakumar, N Metreveli, S Veeranki, S Kundu, and SC Tyagi \\ Department of Physiology and Biophysics, University of Louisville School of Medicine, Louisville, \\ KY-40202
}

\begin{abstract}
Background-Mitochondria constitute $30 \%$ of cell volume and are engaged in two dynamic processes called fusion and fission, regulated by Drp-1(Dynamin related protein) and mitofusin 2 (Mfn2). Previously, we showed that Drp-1 inhibition ameliorates cardiovascular dysfunction following pressure overload in aortic banding model and myocardial infarction. As dynamic organelles, mitochondria are capable of changing their morphology in response to stress. However, whether such changes can alter their function and in turn cellular function is unknown. Further, a direct role of fission and fusion in cardiomyocyte contractility has not yet been studied. In this study, we hypothesize that disrupted fission and fusion balance by increased Drp-1 and decreased Mfn2 expression in cardiomyocytes affect their contractility through alterations in the calcium and potassium concentrations.
\end{abstract}

Methods-To verify this, we used freshly isolated ventricular myocytes from wild type mouse and transfected them with either siRNA to Drp-1 or Mfn2. Myocyte contractility studies were performed by IonOptix using a myopacer. Intracellular calcium and potassium measurements were done using flow cytometry. Immunocytochemistry (ICC) was done to evaluate live cell mitochondria and its membrane potential. Protein expression was done by Western blot and Immunocytochemistry.

Results-We found that silencing mitochondrial fission increased the myocyte contractility, while fusion inhibition decreased contractility with simultaneous changes in calcium and potassium. Also, we observed that increase in fission prompted decrease in Serca-2a and increase in cytochrome c leading to mitophagy.

Conclusion-Our results suggested that regulating mitochondrial fission and fusion have direct effects on overall cardiomyocyte contractility and thus function.

(C) 2015 Published by Elsevier Ireland Ltd.

Please address correspondence to: Srikanth Givvimani M.D; Ph.D., Department of Physiology \& Biophysics, University of Louisville School of Medicine, 500 South Preston Street, Louisville, KY 40202, Phone: 502-852-4425, Fax: 502-852-6239, s0givv01@louisville.edu.

Publisher's Disclaimer: This is a PDF file of an unedited manuscript that has been accepted for publication. As a service to our customers we are providing this early version of the manuscript. The manuscript will undergo copyediting, typesetting, and review of the resulting proof before it is published in its final citable form. Please note that during the production process errors may be discovered which could affect the content, and all legal disclaimers that apply to the journal pertain. 


\section{Introduction}

Cardiovascular disease (CVD) persists as the leading cause of death despite extensive research. The heart is a dynamic organ with abundant mitochondria to meet its continuous energy demands [1]. Mitochondria provide $90 \%$ of ATP and occupy $30 \%$ cell volume, thus becoming an important organelle in adult cardiomyocyte. Rigorous research on cardiac mitochondria has strongly confirmed that structural and functional alterations, termed mitochondrial dynamics play an essential role in maintaining basal cardiac function and any disturbance in these functional changes may lead to various cardiac diseases including myocardial ischemia, infarction and heart failure [2-6]. Mitochondrial dynamics include fission and fusion processes which are balanced under normal physiological status. Aberrant or increased fission will lead to increased mitochondrial fragmentation leading to mitochondrial death or mitophagy. Mitophagy is essential in preventing cell damage during excessive reactive oxygen species (ROS) production. But aberrant mitophagy due to increased mitochondrial fragmentation (fission) leads to cell death and tissue necrosis during disease conditions. We and others have demonstrated abnormal mitophagy in various mice models of cardiovascular disease and regulation of fission process was cardio protective [4, 7-10]. Although 3 fusion proteins and 2 fission proteins are known so far, the predominant among them that are involved in fission and fusion mechanisms are dynamin related protein-1 (Drp-1) and mitofusin 2 (Mfn2) respectively. Several studies have reported that mitochondrial fission by Drp-1 mediates myocardial cell death during ischemia-reperfusion, pressure overload and myocardial infarction [4, 8, 9, 11]. Inhibition of Drp-1 prevented opening of mitochondrial transition pore and reduced infarct size in mouse coronary artery ligation [9]. It was also shown that Drp-1 induced mitochondrial fragmentation precedes ROS production in ventricular myocytes during increased cytosolic calcium [12]. Preclinical studies have effectively demonstrated that Mdivi-1 (mitochondrial division inhibitor), a Drp-1 specific inhibitor is protective in various cardiac diseases. Mdivi-1, by inhibiting Drp-1 preserved the mitochondrial morphology, reduced cytosolic calcium and prevented cell death during ischemia reperfusion [10]. It was also reported that Drp-1 mediates cardiac hypertrophy during pressure overload conditions and treatment with Mdivi-1 attenuates this process $[4,13]$. Mitochondrial fusion protein (Mfn2) regulates mitochondrial structure and metabolism, while its expression is decreased in diabetes and obesity, it is improved with weight loss and exercise [14, 15]. Mfn2 is primarily involved in mitochondrial calcium reuptake mechanism necessary for ATP production. Mfn2 is localized in endoplasmic reticulum (ER) and regulates ER structure function and calcium uptake [16]. Studies have reported that Mfn2 expression is decreased in various rat models of cardiac hypertrophy including spontaneous hypertensive rats, transverse aortic banding and myocardial infarction [17].

Although, it was known that disrupted mitochondrial fission-fusion balance with increased fragmentation is detrimental to the cell leading to mitophagy its direct effect on cardiomyocyte contractility is unclear. Thus in this study we hypothesize that modulating mitochondrial fission-fusion balance will interfere directly in the cardiomyocyte contractility through alterations in the cytosolic calcium and potassium concentrations. 


\section{Methods}

\section{Isolation of ventricular cardiomyocytes}

Fresh perfusion buffer (120.4 mM NaCl, $14.7 \mathrm{mM} \mathrm{KCl}, 0.6 \mathrm{mM} \mathrm{KH} 2 \mathrm{PO} 4,0.6 \mathrm{mM}$ Na2HPO4, 10 mM Na-HEPES, 1.2 mM MgSO4+-7H2O, 4.6 mM NaHCO3, 30 mM Tuarine, $10 \mathrm{mM}$ BDM, $5.5 \mathrm{mM}$ Glucose; $\mathrm{pH}$ 7.0) prepared daily. The perfusion system is prepared as follows: the temperature of the circulation water bath is set so that the temperature of the outflow liquid at the tip of the cannula (20-g needle with the nub filed flat and smooth) is $37^{\circ} \mathrm{C}$. Flow rate is set at $4.0 \mathrm{ml} / \mathrm{min}$ prior to the cardiomyocyte isolation, about $100 \mathrm{ml}$ of distilled water is run through the system. Then the system is perfused with perfusion buffer for at least 5 minutes.

A mouse of at least 20 grams is injected with heparin (1000 U/kg; i.p.). Then the mouse is anesthetized with TBE (Avertin $100 \mathrm{mg} / \mathrm{kg}$, i.p.). The chest is wiped with $70 \%$ ethanol. A skin incision is made revealing the xiphoid process. The rib cage is completely cut starting at the xiphoid process running up the chest cavity. To avoid heart damage the diaphragm is cut as well. The heart is secured with forceps and all vessels are cut. The aorta is cut so as to leave the maximal length, which is important for rapid cannulation. The dissected heart is immediately placed in a Petri dish containing ice-cold calcium free perfusion buffer. To expose the aorta, all the remnant excess tissue is removed and discarded. Holding the aorta with two fine forceps, it is slid onto the vertically mounted cannula until the tip of the needle reaches the aortic valve. The heart is secured on the needle with a small brass clip and is immediately perfused with perfusion buffer at a flow rate of $4.0 \mathrm{ml} / \mathrm{min}$ for $4 \mathrm{~min}$ or until the outflow from apex is clear from blood. The aorta is tied to the needle with silk thread. Then the perfusion with digestion buffer consisting of $29 \mathrm{ml}$ perfusion buffer and $1.0 \mathrm{ml}$ of 5 $\mathrm{mg} / \mathrm{ml}$ Liberase TH Research Grade (Roche Diagnostics Corp. Indianapolis, IN) is continued for 7-8 min. At the end of the perfusion the tissue became soft, swollen and light pink.

After the perfusion the heart is cut from the needle just below the atria using sterile fine scissors and is placed in a Petri dish with $10 \mathrm{ml}$ incubation buffer $(135 \mathrm{mM} \mathrm{NaCl}, 4 \mathrm{mM}$ $\mathrm{KCl}, 1 \mathrm{mM} \mathrm{MgCl} 2,10 \mathrm{mM}$ HEPES, $0.33 \mathrm{mM}$ NaH2PO4, 10mM glucose, 10mM BDM, $1.2 \mathrm{mM} \mathrm{CaCl} 2$ ). The heart is gently teased into small pieces with fine forceps. The obtained suspension of cardiomiocytes are gently pipetted up and down with a plastic pipette ( $2 \mathrm{~mm}$ tip) several times. Then the cells are transferred to a $60-\mathrm{mm}$ nonstick Valmark dish and 10 , $20,30,30$ and $30 \mu \mathrm{l}$ of $100 \mathrm{mM} \mathrm{CaCl} 2$ solution is added at $5 \mathrm{~min}$ intervals. The final content of calcium is $1.2 \mathrm{mM}$. Isolated myocytes are maintained at room temperature in contractility buffer (Minimum Essential Medium with Hanks Salts (Gibco), Butadione Monoxime (25 $\mathrm{mM}$, Sigma), Bovine Calf Serum (5\%, HyClone), Penicillin (100u/ml, Sigma), L-Glutamine (2 mM, Gibco).

\section{Analysis of myocyte contractility}

Cardiomyocyte contractility is controlled by electrical stimulation. Mechanical properties of isolated ventricular myocytes are assessed by video-based edge detection. An inverted 
microscope, a low light-level video camera and a computer-based motion analyzer are used to track the movement of cell edges (6).

The isolated myocytes are diluted approximately ten fold with contractility buffer and placed on a Teflon glass coverslip dish mounted on the stage of an inverted microscope (Olympus, IX-70). Then the cells are field-stimulated to contract by the MyoPacer field stimulator through a pair of platinum electrodes at a frequency of $1.0 \mathrm{~Hz}$, pulse duration of 4 $\mathrm{ms}$ and amplitude of 10 volts. The image of the myocyte is obtained with an IonOptix Myo Cam camera side-mounted onto the microscope and displayed on a computer monitor using the Soft-Edge software. Typically 5 individual myocytes are recorded before replacing the myocytes with a fresh batch of unstimulated myocytes. This prevents exhaustion of the myocytes due to prolonged stimulation. Twenty myocytes are analyzed from each heart. The cells being studied are scanned every $8.3 \mathrm{~ms}$ so that the amplitude and velocity of shortening and lengthening can be recorded with high fidelity. The displacements of cell edges at both ends of the myocyte are detected and converted to an analog voltage signal, which is then digitized and stored for off-line analysis. Steady-state twitches (15--20) are analyzed for cell length changes using the Soft-Edge software and averaged for each myocyte. Cell shortening and re-lengthening are assessed by percentage of peak shortening (\%PS), time to 90\% peak shortening (TPS90), time to 90\% re-lengthening (TR90), and maximal velocities of shortening and re-lengthening $( \pm \mathrm{dL} / \mathrm{dt})$.

\section{Intracellular fluorescence measurement of $\mathrm{Ca} 2+$}

A separate cohort of myocytes is loaded with Fura 2-AM $(1.0 \mu \mathrm{mol} / \mathrm{l})$ for $30 \mathrm{~min}$, and fluorescence measurements are recorded with a dual-excitation fluorescence photomultiplier system (IonOptix). Myocytes are placed in a dish chamber on the stage of an Olympus IX-70 inverted microscope and imaged through a Fluor 100 $\times$ oil objective. Cells are exposed to light emitted by a 75-W lamp and passed through either a 360- or a 380-nm filter (bandwidths are $\pm 15 \mathrm{~nm}$ ), while being stimulated to contract at $1.0 \mathrm{~Hz}$. Fluorescence emissions are detected between 480 and $520 \mathrm{~nm}$ by a photomultiplier tube after first illuminating the cells at $360 \mathrm{~nm}$ for $0.5 \mathrm{~s}$ and then at $380 \mathrm{~nm}$ for the duration of the recording protocol (333-Hz sampling rate). The 360-nm excitation scan is repeated at the end of the protocol, and qualitative changes in intracellular $\mathrm{Ca} 2+$ concentration $([\mathrm{Ca} 2+] \mathrm{i})$ are inferred from the ratio. Intracellular $\mathrm{Ca} 2+$ transients are measured as changes of Fura 2AM fluorescence intensity (FFI). $\Delta \mathrm{FFI}$ is determined as the difference between the levels of $\mathrm{Ca} 2+$ in systolic and diastolic conditions $(\Delta \mathrm{FFI}=$ peak FFI - baseline FFI). The time course of the fluorescence signal decay ( $\tau$ : the duration where $\mathrm{Ca} 2+$ transient decays $67 \%$ from the peak level) is calculated to assess intracellular $\mathrm{Ca} 2+$ clearing rate.

Transfection of myocytes-After isolation of ventricular myocytes, myocytes are transfected overnight in an incubator with either siRNA to Drp-1 or Mfn2 (300 ng/100 $\mu \mathrm{l}$ siRNA from each of four different oligonucleotides) in a 6 well plate using $12 \mu \mathrm{l}$ of Qiagen Hi-perfect transfection reagent following manufacturer's transfection protocol and compared with control myocytes. Validation of transfection is done with GFP negative control and also by performing immunocytochemistry for Drp-1 and Mfn2 (Figure 1). 
Flow cytometry-The heart was digested as described above in isolation of ventricular myocytes to obtain single-cell suspension of cardiomyocytes. The cells were incubated with Fura-2-acetoxymethyl ester, Fura2-AM, $(20 \mu \mathrm{g} / \mathrm{mL})$ and potassium-binding benzofuran isophthalate acetoxymethyl ester, PBFI, $(20 \mu \mathrm{g} / \mathrm{mL})$ at $37^{\circ} \mathrm{C}$ for $30 \mathrm{~min}$. 7AAD stain was used for cell viability. Data was acquired using BD AccuriTM C6 flow cytometer and analyzed with C6 analysis software.

Western blotting and Antibodies-Western blotting with the cell lysates from the cardiomyocytes were performed as described before [18]. Briefly, $\sim 30$ ug of cell lysates were resolved on polyacrylamide gel and were blotted overnight to PVDF membranes. After probing with appropriate primary and secondary antibodies, membranes were developed using Bio-Rad ChemiDoc-XRS+ instrument equipped with image lab software (Hercules, $\mathrm{CA}$ ). Images were processed and relative abundance of proteins of interest was quantified after normalization to loading control (GAPDH levels). The antibodies: anti-Cytochrome C and anti-SERCA2 ATPase are from Abcam (Cambridge, MA), anti-potassium channel KvLQT1 and anti-GAPDH are from Millipore (Billerica, MA).

\section{Results}

\section{Altered fission and fusion balance affects the myocyte contractility through changes in cytosolic calcium}

Freshly isolated mouse ventricular myocytes were overnight transfected with either Drp-1 or Mfn2 siRNA and their contractility was measured by video based microscope platform, IonOptix using stimulation of $1 \mathrm{~Hz}$ frequency. While transfecting myocytes with Drp-1 siRNA results in improved contractility as shown by increased peak shortening tracing (Figure 2A) compared to control, we noticed decreased contractility with Mfn2 siRNA transfection. Similarly, the departure and return velocities from peak shortening tracings also showed increased contractility in Drp-1 siRNA myocytes compared to Mfn2 siRNA myocytes (Figure 2B). Our results suggested that regulating mitochondrial fission with Drp-1 siRNA augmented myocyte contractility and interestingly, the contractility was diminished with Mfn2 siRNA signifying the role of fusion in myocyte contractility.

We also measured cytosolic calcium through IonOptix using Fura 2-AM dye from Invitrogen. Although we didn't measure the exact intracellular calcium levels, we presented the calcium transient recordings derived from the ratio of Fura to Fluorescence intensity $(\Delta \mathrm{FFI})$. We observed that calcium transient decay curve tracings were increased (suggesting increased cytosolic calcium) in the myocytes transfected with Mfn2 siRNA whereas the decay curves were decreased (suggesting decreased cytosolic calcium) in myocytes that were transfected with Drp-1 siRNA (Figure 2C). Tau index that suggests relaxation or recovery phase of cardiomyocytes was also increased in Mfn2 siRNA myocytes and decreased in Drp-1 siRNA compared to non transfected control group of myocytes (Figure 2D). Additionally we confirmed the changes in cytosolic calcium by performing both flow cytometry studies with Fura 2-AM (Figure 3A) and immunocytochemistry with calcium sensitive dye (Rhod-2, AM, Molecular probes) that emits increased fluorescence upon binding to calcium (Figure 4). Above findings suggest that there was decreased or basal 
level of cytosolic calcium during Drp-1 silencing (increased mitochondrial fusion) and there was excessive cytosolic calcium during Mfn2 silencing (increased mitochondrial fission) which could result in calcium induced cell damage or death.

\section{siRNA to Drp-1 resulted in decreased potassium channels and increased cytosolic potassium}

Potassium $\left(\mathrm{K}^{+}\right)$channels and cytosolic potassium play in important role in myocyte action potential duration. We observed that isolated ventricular myocytes after transfection with Mfn2 siRNA showed increased expression of $\mathrm{K}^{+}$channels and after Drp-1 siRNA transfection, these channels are decreased in their expression (Figure 5A). To verify the effect of $\mathrm{K}^{+}$channels on the cytosolic potassium, we performed flow cytometry and immunocytochemistry using $\mathrm{K}^{+}$sensitive PBFI dye from molecular probes. Interestingly, we found that cytosolic potassium was decreased in myocytes that were transfected with Mfn2 siRNA while it was increased in those myocytes that were transfected with Drp-1 siRNA compared to non transfected myocytes (Figure 3B, 5B \& 8). Our results suggest that by inhibition of mitochondrial fission, there was increased cytosolic potassium that could lead to increased action potential duration.

\section{Increased mitochondrial fission leads to increase in cytochrome c leakage}

Previous studies demonstrated that mitochondria play a key role in regulating autophagy. The release of cytochrome $\mathrm{c}$ from mitochondria into cytosol results in activation of caspases leading to cell death. We verified the role of mitochondrial fusion and fission relation to cell death by measuring cytochrome $\mathrm{c}$ in isolated cardiomyocytes that were transfected with either siRNA to Drp-1 or siRNA to Mfn2. We observed that in Mfn2 siRNA transfected myocytes, in which fusion is silenced with uninterrupted fission process there was significant increase in cytochrome c leakage (Figure $6 \& 8$ ). Interestingly, we noticed that there was decreased release of cytochrome $\mathrm{c}$ in Drp-1 siRNA transfected myocytes. Our results suggested that exacerbated mitochondrial fission or fragmentation could stimulate increase in cytochrome $\mathrm{c}$ leakage leading to cell death.

\section{Serca-2a expression was decreased in cardiomyocytes transfected with Mfn2 siRNA}

While sarco/endoplasmic reticulum $\mathrm{Ca}^{2+}$ ATPase (Serca) is essential in calcium reuptake mechanism to transfer calcium from cytosol to sarco/endoplasmic reticulum, Mfn2 acts as a uniporter to pump back excess calcium into mitochondria. As our previous results suggested increase in cytosolic calcium during Mfn2 silencing, we thought of measuring Serca levels in our experiment. Results from immunocytochemistry and western blot suggest that there was decreased Serca-2a expression in cardiomyocytes that were transfected with Mfn2 siRNA (Figure $7 \&$ 8). Interestingly, Serca-2a expression was increased in myocytes that were transfected with Drp1 siRNA. Our findings suggest that decreased Mfn2 or increased mitochondrial fission lead to excessive accumulation of cytosolic calcium which could be detrimental to the cell viability. 


\section{Discussion}

The essential role of mitochondria in cardiac pathology has been demonstrated by various researchers for more than a decade. Although studies have demonstrated the beneficial effects of Drp-1(mitochondrial fission protein) inhibition and cardio protective role of mitochondrial fusion protein Mfn2 in various preclinical disease models, their direct role in the cardiomyocyte contractility or cellular changes due to imbalance of fission and fusion proteins has not been verified. Here in this study we hypothesize that modulating mitochondrial fission-fusion balance will interfere in the cardiomyocyte contractility through alterations in the cytosolic calcium and potassium concentrations.

Although calcium is required for cardiomyocyte contractility, cytosolic calcium overload leads to cellular injury and death [19]. Mitochondria are the important cytoplasmic calcium regulators similar to sarco/endoplasmic reticulum and mitochondrial calcium overload lead to irreversible cellular damage and death during cardiac pathology [20]. It was reported that mitochondrial damage results in disruption of oxidative phosphorylation generating excess reactive oxygen and nitrogen species and a reduction in ATP production, disruption of $\mathrm{Ca} 2+$ homeostasis and also triggers apoptosis [21,22]. We have shown previously that mitochondrial division inhibitor (Mdivi-1) ameliorates ventricular dysfunction following pressure overload injury [4]. Studies have also reported that Drp1 expression is increased during ischemia-reperfusion injury and inhibition of Drp1 by Mdivi-1 attenuates the injury and improves cardiomyocyte contractility [10]. Similarly our current results also demonstrate increased myocyte contractility (Figure 2A \& 2B) by inhibition of mitochondrial fission by Drp1 siRNA. Conversely, inhibition of mitochondrial fusion by Mfn2 siRNA decreased myocyte contractility (Figure 2A \& 2B). It is interesting that we observed the cytosolic calcium changes by interrupting mitochondrial fission (Drp1) and fusion (Mfn2) proteins with their respective siRNA transfection. We found that by inhibiting Mfn2, there was increased accumulation of cytosolic calcium (Figure 3A, $4 \& 8$ ) that could be detrimental to cardiomyocyte. This is consistent with previous findings that Mfn2 deletion would ruin the sarcoplasmic reticulum and mitochondrial coupling that results in decreased calcium transfer to mitochondria and impairs Krebs cycle, which is of significant energy source in the heart [23].

Opening of potassium channels and potassium efflux is responsible for the depolarization phase during cardiac action potential. Previously, it was reported that, commercially available Drp-1 inhibitor, Mdivi-1(mitochondrial division inhibitor) inhibited potassium channels in the heart [24]. Although, they suggested that the inhibition of $\mathrm{K}^{+}$channels by Mdivi-1 is a direct effect, here we observed that by silencing Drp-1 with siRNA, potassium channels were decreased (Figure 5A \& 8). Interestingly, we also found that intracellular potassium levels were also increased accordingly (Figure 3B \& 5B) that could be responsible for increased cardiac action potential duration during inhibition of mitochondrial fission. Contrary to this we observed opposite results while inhibiting the mitochondrial fusion by silencing Mfn2 with siRNA.

Mitochondrial proteins play an important role in cell death pathways. It was reported that preventing mitochondrial fission by inhibiting fission protein Drp-1 delays or inhibits 
apoptotic markers like cytochrome c leakage and thereby cell death [25-27]. Similarly, we observed that there is excessive cytochrome c leakage when there is increased fission (Mfn2 siRNA group) and decreased during Drp-1 inhibition (Figure $6 \& 8$ ).

Calcium recycling is key for effective cardiac relaxation. Failure to recycle calcium results in overload and impaired cardiac function leading ultimately to cell death. Sarco endoplasmic reticulum (SR) calcium ATPase (Serca) is key enzyme in recycling calcium in SR. The cytosolic calcium alterations in turn cardiac myocyte contractility changes due to mitochondrial proteins made us to evaluate Serca levels in our experiment. It was reported that Mfn2 plays an important role in mitochondrial calcium reuptake and ATP production [16]. Our observation of increased cytosolic calcium during Mfn2 inhibition with siRNA is supported by decreased Serca levels and vice versa in Drp-1 inhibition (Figure 7).

In conclusion, our findings for the first time demonstrated that changes in mitochondrial fission and fusion proteins directly affect the myocyte contractility with changes in cytosolic calcium and potassium levels. We also observed that increased mitochondrial fission resulted in increased cytosolic calcium, increased cytochrome c leakage and decreased Serca levels, which is detrimental to cell.

\section{Acknowledgments}

A part of this study was supported by NIH grants: HL-74185, NS-084823 and HL-108621.

\section{REFERENCES}

[1]. Hom J, Sheu SS. Morphological dynamics of mitochondria--a special emphasis on cardiac muscle cells. Journal of molecular and cellular cardiology. 2009; 46:811-820. [PubMed: 19281816]

[2]. Ducharme A, Frantz S, Aikawa M, Rabkin E, Lindsey M, Rohde LE, Schoen FJ, Kelly RA, Werb Z, Libby P, Lee RT. Targeted deletion of matrix metalloproteinase- 9 attenuates left ventricular enlargement and collagen accumulation after experimental myocardial infarction. J.Clin.Invest. 2000; 106:55-62. [PubMed: 10880048]

[3]. Friehs I, Margossian RE, Moran AM, Cao-Danh H, Moses MA, del Nido PJ. Vascular endothelial growth factor delays onset of failure in pressure-overload hypertrophy through matrix metalloproteinase activation and angiogenesis. Basic Res.Cardiol. 2006; 101:204-213. [PubMed: 16369727]

[4]. Givvimani S, Munjal C, Tyagi N, Sen U, Metreveli N, Tyagi SC. Mitochondrial division/ mitophagy inhibitor (Mdivi) ameliorates pressure overload induced heart failure. PloS one. 2012; 7:e32388. [PubMed: 22479323]

[5]. Moshal KS, Tyagi N, Moss V, Henderson B, Steed M, Ovechkin A, Aru GM, Tyagi SC. Early induction of matrix metalloproteinase- 9 transduces signaling in human heart end stage failure. J.Cell Mol.Med. 2005; 9:704-713. [PubMed: 16202218]

[6]. Peterson JT, Hallak H, Johnson L, Li H, O’Brien PM, Sliskovic DR, Bocan TM, Coker ML, Etoh T, Spinale FG. Matrix metalloproteinase inhibition attenuates left ventricular remodeling and dysfunction in a rat model of progressive heart failure. Circulation. 2001; 103:2303-2309. [PubMed: 11342481]

[7]. Brooks C, Wei Q, Cho SG, Dong Z. Regulation of mitochondrial dynamics in acute kidney injury in cell culture and rodent models. The Journal of clinical investigation. 2009; 119:1275-1285. [PubMed: 19349686]

[8]. Gharanei M, Hussain A, Janneh O, Maddock HL. 15 Mitochondrial Division Inhibitor-1 Protects against Doxorubicin-Induced Cardiotoxicity. Heart. 2014; 100(Suppl 1):A6. 
[9]. Ong SB, Subrayan S, Lim SY, Yellon DM, Davidson SM, Hausenloy DJ. Inhibiting mitochondrial fission protects the heart against ischemia/reperfusion injury. Circulation. 2010; 121:2012-2022. [PubMed: 20421521]

[10]. Sharp WW, Fang YH, Han M, Zhang HJ, Hong Z, Banathy A, Morrow E, Ryan JJ, Archer SL. Dynamin-related protein 1 (Drp1)-mediated diastolic dysfunction in myocardial ischemiareperfusion injury: therapeutic benefits of Drp1 inhibition to reduce mitochondrial fission. FASEB journal : official publication of the Federation of American Societies for Experimental Biology. 2014; 28:316-326. [PubMed: 24076965]

[11]. Disatnik MH, Ferreira JC, Campos JC, Gomes KS, Dourado PM, Qi X, Mochly-Rosen D. Acute inhibition of excessive mitochondrial fission after myocardial infarction prevents long-term cardiac dysfunction. Journal of the American Heart Association. 2013; 2:e00461. [PubMed: 24103571]

[12]. Hom J, Yu T, Yoon Y, Porter G, Sheu SS. Regulation of mitochondrial fission by intracellular $\mathrm{Ca} 2+$ in rat ventricular myocytes. Biochimica et biophysica acta. 2010; 1797:913-921. [PubMed: 20347716]

[13]. Chang YW, Chang YT, Wang Q, Lin JJ, Chen YJ, Chen CC. Quantitative phosphoproteomic study of pressure-overloaded mouse heart reveals dynamin-related protein 1 as a modulator of cardiac hypertrophy. Molecular \& cellular proteomics: MCP. 2013; 12:3094-3107. [PubMed: 23882026]

[14]. Bach D, Naon D, Pich S, Soriano FX, Vega N, Rieusset J, Laville M, Guillet C, Boirie Y, Wallberg-Henriksson H, Manco M, Calvani M, Castagneto M, Palacin M, Mingrone G, Zierath JR, Vidal H, Zorzano A. Expression of Mfn2, the Charcot-Marie-Tooth neuropathy type 2A gene, in human skeletal muscle: effects of type 2 diabetes, obesity, weight loss, and the regulatory role of tumor necrosis factor alpha and interleukin-6. Diabetes. 2005; 54:2685-2693. [PubMed: 16123358]

[15]. Cartoni R, Leger B, Hock MB, Praz M, Crettenand A, Pich S, Ziltener JL, Luthi F, Deriaz O, Zorzano A, Gobelet C, Kralli A, Russell AP. Mitofusins 1/2 and ERRalpha expression are increased in human skeletal muscle after physical exercise. The Journal of physiology. 2005; 567:349-358. [PubMed: 15961417]

[16]. de Brito OM, Scorrano L. Mitofusin 2 tethers endoplasmic reticulum to mitochondria. Nature. 2008; 456:605-610. [PubMed: 19052620]

[17]. Fang L, Moore XL, Gao XM, Dart AM, Lim YL, Du XJ. Down-regulation of mitofusin-2 expression in cardiac hypertrophy in vitro and in vivo. Life sciences. 2007; 80:2154-2160. [PubMed: 17499311]

[18]. Veeranki S, Givvimani S, Pushpakumar S, Tyagi SC. Hyperhomocysteinemia attenuates angiogenesis through reduction of HIF-1alpha and PGC-1alpha levels in muscle fibers during hindlimb ischemia. American journal of physiology. Heart and circulatory physiology. 2014; 306:H1116-1127. [PubMed: 24585779]

[19]. Marsh JD, Smith TS. Calcium overload and ischemic myocardial injury. Circulation. 1991; 83:709-711. [PubMed: 1991389]

[20]. Shen AC, Jennings RB. Myocardial calcium and magnesium in acute ischemic injury. The American journal of pathology. 1972; 67:417-440. [PubMed: 5033257]

[21]. Diaz F, Moraes CT. Mitochondrial biogenesis and turnover. Cell calcium. 2008; 44:24-35. [PubMed: 18395251]

[22]. Figueira TR, Barros MH, Camargo AA, Castilho RF, Ferreira JC, Kowaltowski AJ, Sluse FE, Souza-Pinto NC, Vercesi AE. Mitochondria as a source of reactive oxygen and nitrogen species: from molecular mechanisms to human health. Antioxidants \& redox signaling. 2013; 18:20292074. [PubMed: 23244576]

[23]. Chen Y, Liu Y, Dorn GW 2nd. Mitochondrial fusion is essential for organelle function and cardiac homeostasis. Circulation research. 2011; 109:1327-1331. [PubMed: 22052916]

[24]. So EC, Hsing CH, Liang CH, Wu SN. The actions of mdivi-1, an inhibitor of mitochondrial fission, on rapidly activating delayed-rectifier $\mathrm{K}(+)$ current and membrane potential in HL-1 murine atrial cardiomyocytes. European journal of pharmacology. 2012; 683:1-9. [PubMed: 22374256] 
[25]. Yu T, Fox RJ, Burwell LS, Yoon Y. Regulation of mitochondrial fission and apoptosis by the mitochondrial outer membrane protein hFis1. Journal of cell science. 2005; 118:4141-4151. [PubMed: 16118244]

[26]. Lee YJ, Jeong SY, Karbowski M, Smith CL, Youle RJ. Roles of the mammalian mitochondrial fission and fusion mediators Fis1, Drp1, and Opa1 in apoptosis. Molecular biology of the cell. 2004; 15:5001-5011. [PubMed: 15356267]

[27]. Frank S, Gaume B, Bergmann-Leitner ES, Leitner WW, Robert EG, Catez F, Smith CL, Youle RJ. The role of dynamin-related protein 1, a mediator of mitochondrial fission, in apoptosis. Developmental cell. 2001; 1:515-525. [PubMed: 11703942] 
Transfection validity
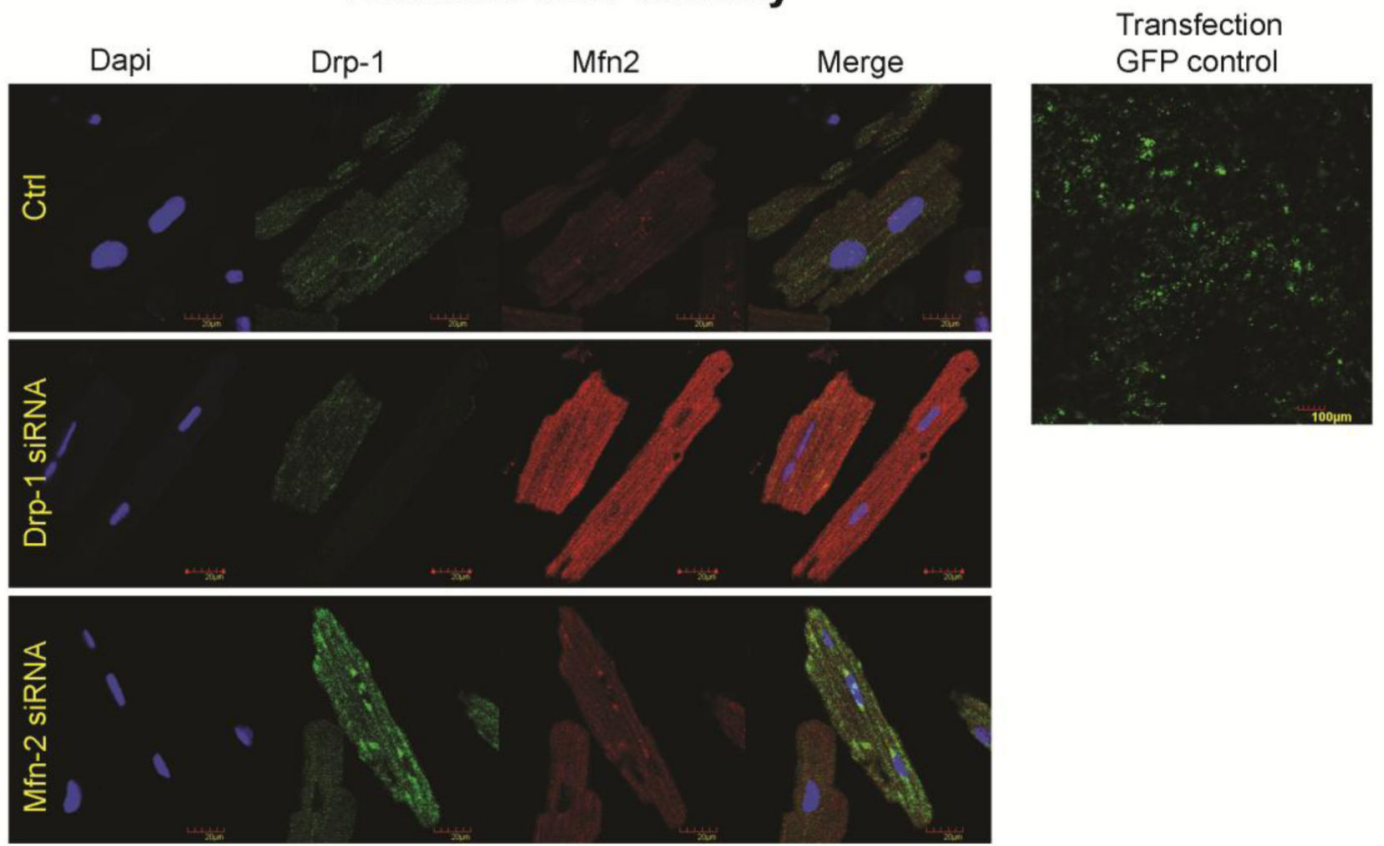

Figure 1.

Transfection after overnight is validated with GFP negative control and followed by the immunocytochemistry staining for the silenced genes Drp-1(green), Mfn2 (red) and dapi (blue) staining nuclei. We observed that during transfection with Drp-1 siRNA, we noticed least expression of it while we found increased expression of fusion protein Mfn2. Similarly, transfection of cardiomyocytes with Mfn2 siRNA resulted in decreased expression of Mfn2 suggesting decreased fusion process, while that of Drp- 1 is increased suggesting increased fission process. 
A

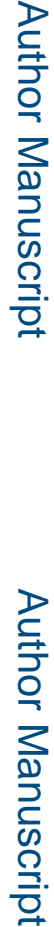

B

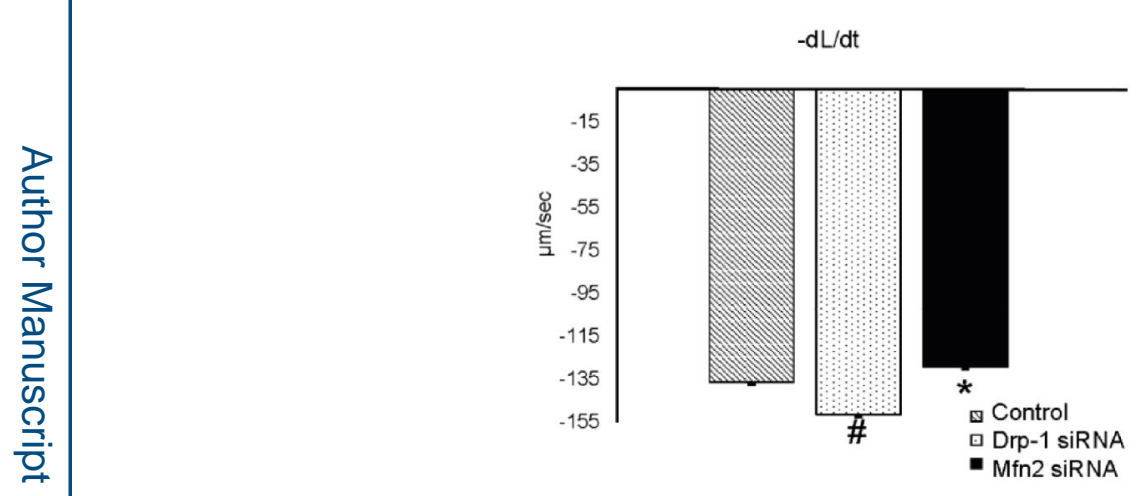

Cardiomyocyte Contractility peaks

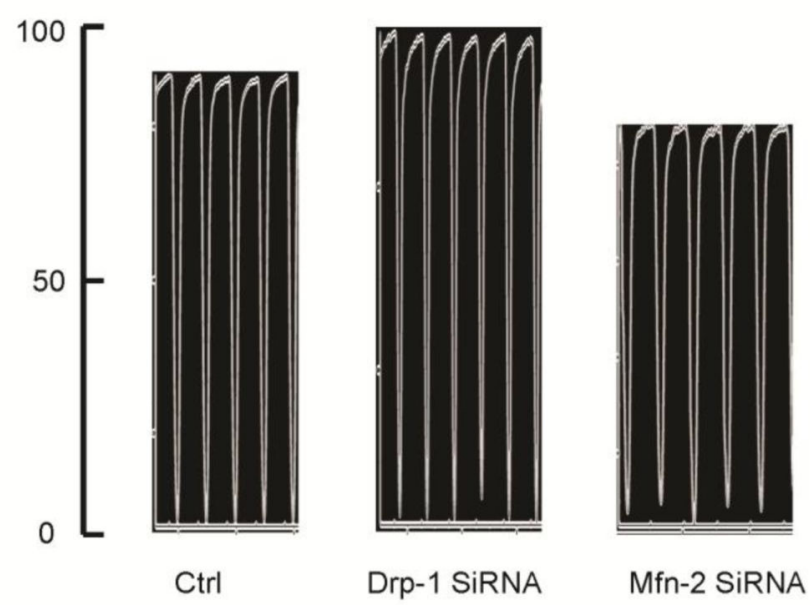

Changes in peak shortening and lengthening

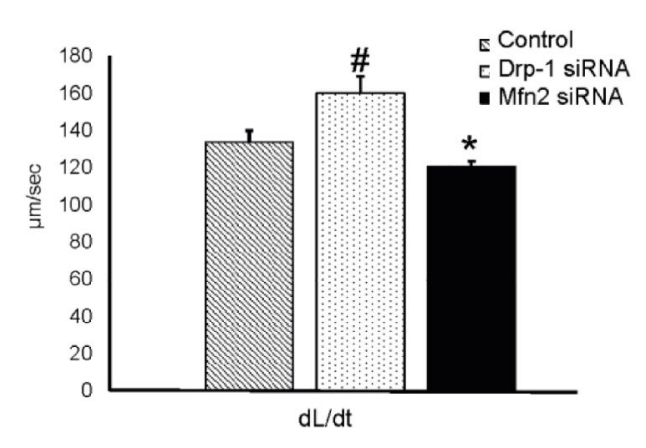

글

Int J Cardiol. Author manuscript; available in PMC 2016 May 06. 
c

\section{$\mathrm{Ca}^{+2}$ transient decay curves}
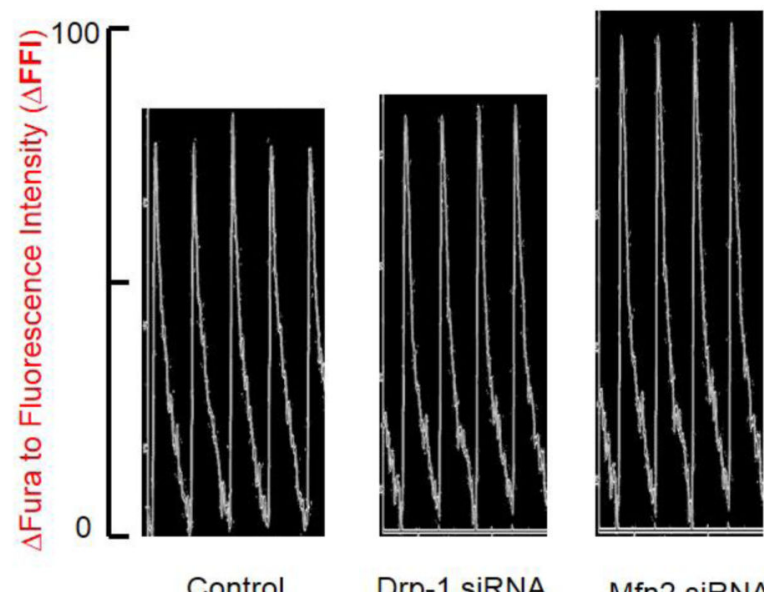

Control

Drp-1 siRNA

Mfn2 siRNA

$\overline{5 \mathrm{msec}}$

\section{D}

\section{Myocyte relaxation}

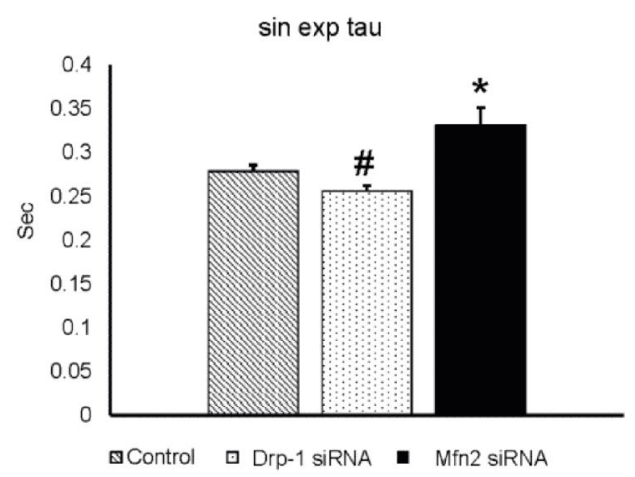

Figure 2.

A) Representative contractility tracings measured by video based microscope platform (Ionoptix, Beverley, MA) from control, Drp-1 siRNA and Mfn2 siRNA group of myocytes. B) Rate of peak shortening and lengthening of cardiomyocyte. C) Representation of $\mathrm{Ca} 2+$ decay curves and $\mathbf{D}$ ) bar graph represent recovery or relaxation phase of cardiomyocytes. Data represents mean \pm SE from $n=5$ per group; * $p \leq 0.05$ compared to control and Drp- 1 siRNA; \#p $\leq 0.05$ compared control. 


\section{Flow cytometry data}

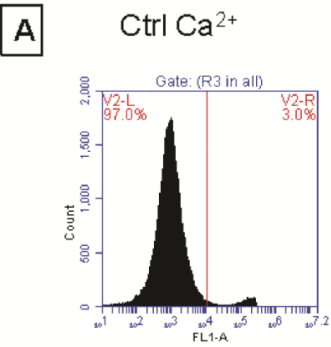

B $\quad \mathrm{Ctrl} \mathrm{K}^{+}$

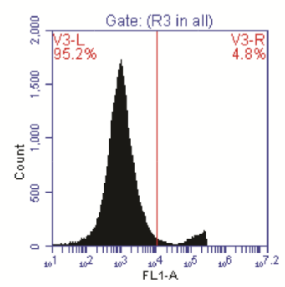

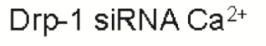

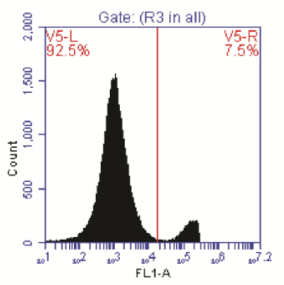

Drp-1 siRNA K ${ }^{+}$

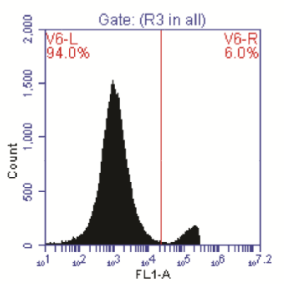

Mfn2 siRNA Ca ${ }^{2+}$
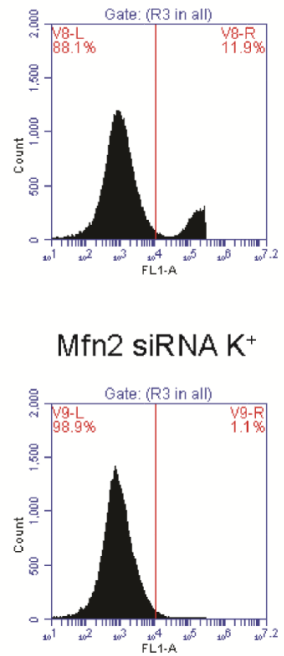

Ctrl $\mathrm{Ca}^{2+}$

- Drp-1 siRNA Ca ${ }^{2+}$

- Mfn2 siRNA Ca ${ }^{2+}$
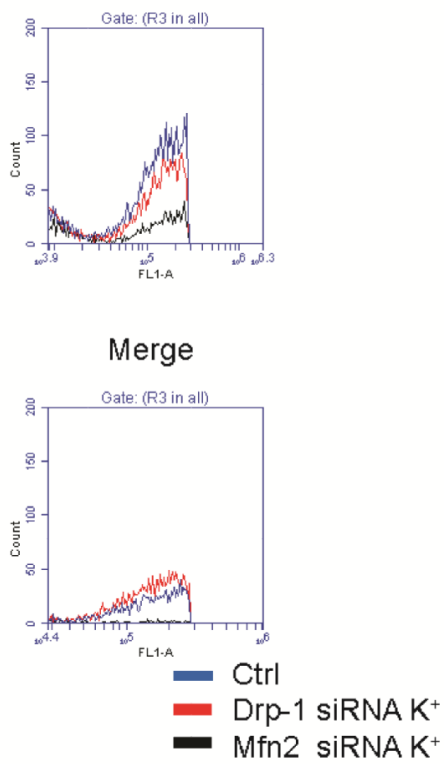

Figure 3.

A) Flow cytometry data representing cytosolic calcium labelled with Invitrogen fura2-AM from control, Drp-1 siRNA and Mfn2 siRNA group of myocytes and B) data representing cytosolic potassium labelled with Invitrogen PBFI-AM dye. 7 AAD stain was used to exclude dead cells. 
ICC images of Intracellular calcium
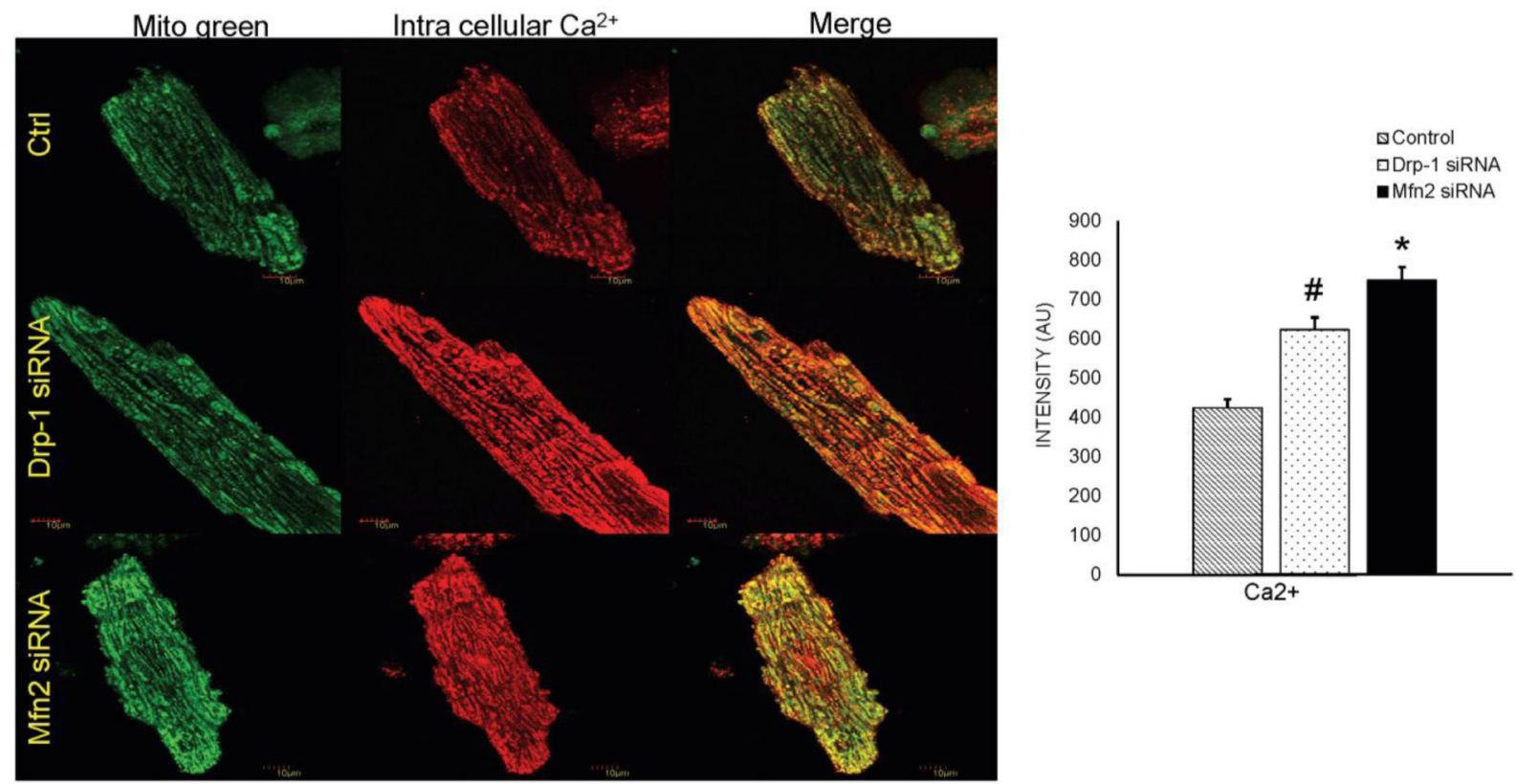

Figure 4.

Immunocytochemistry images of cardiomyocytes from control, Drp-1 siRNA and Mfn2 siRNA group stained with calcium sensitive dye (Rhod-2, AM, Molecular probes) that emits red fluorescence upon binding to calcium and Mito tracker green to label mitochondria with green fluorescence and dapi (blue) staining representing nuclei. Data represents mean $\pm \mathrm{SE}$ from $\mathrm{n}=5$ per group; ${ }^{*} \mathrm{p} \leq 0.05$ compared to control and Drp-1 siRNA; \#p $\leq 0.05$ compared control. 

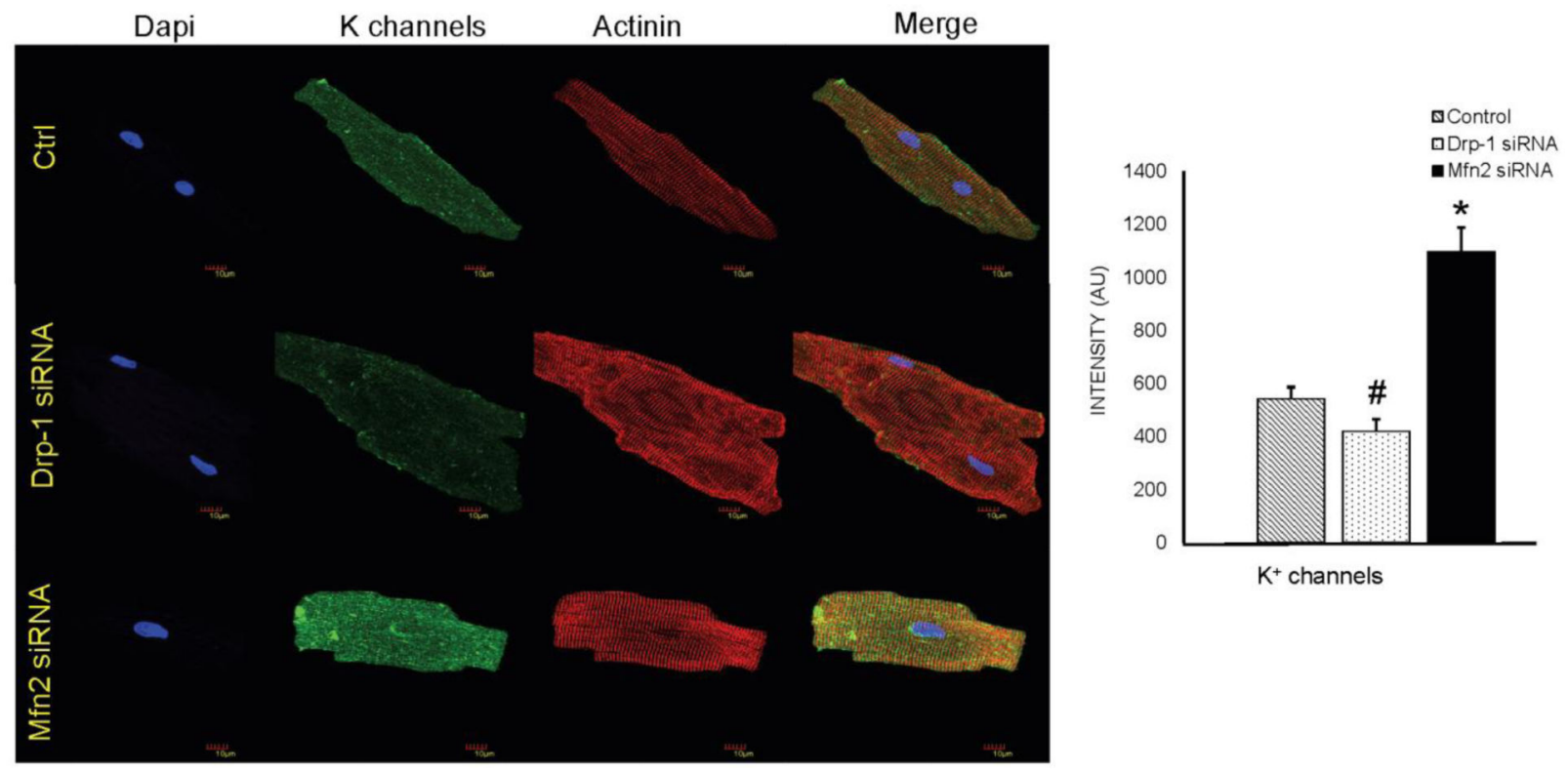

B

ICC images of Intracellular potassium
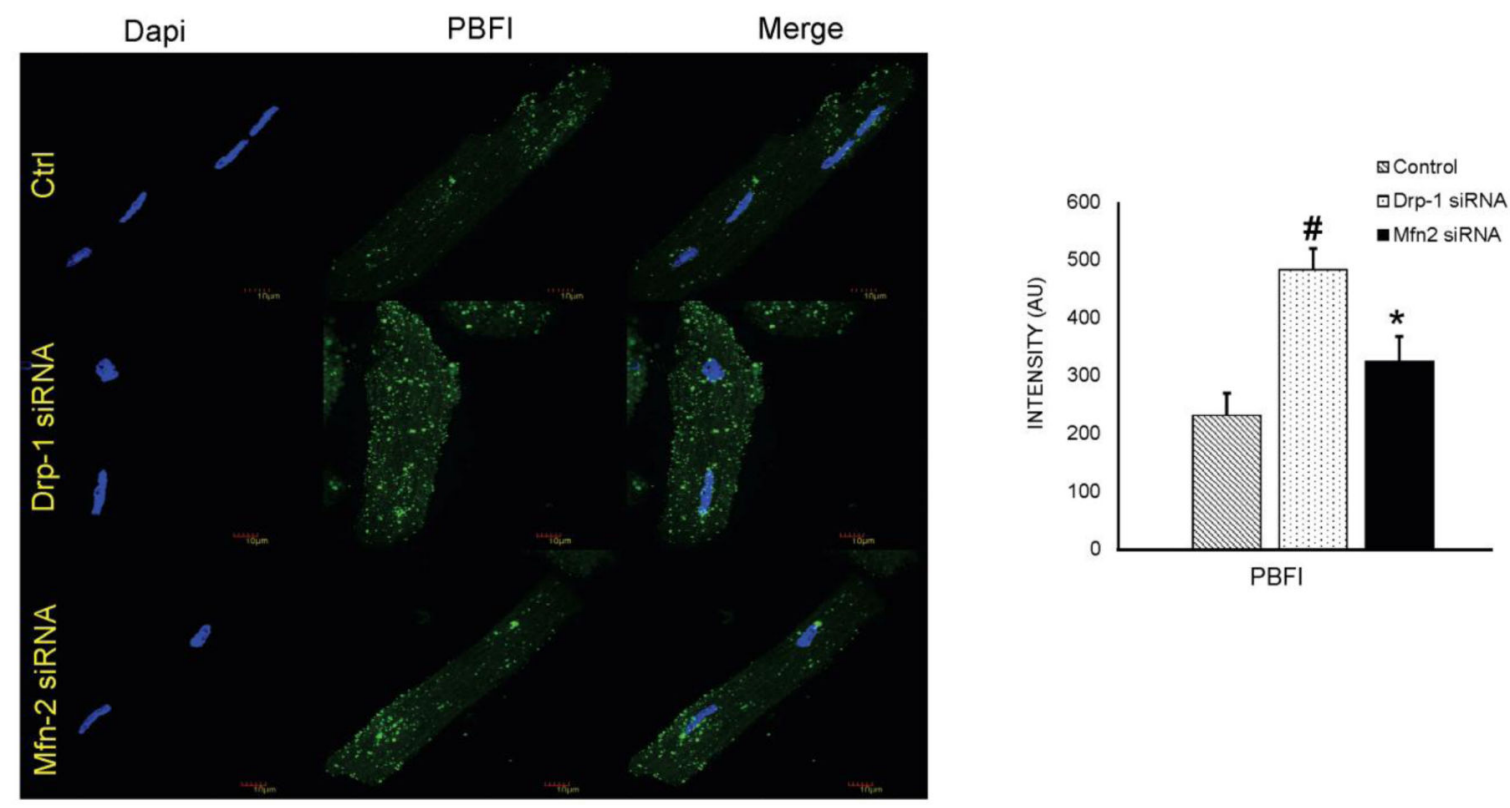

Figure 5.

A) Immunocytochemistry images of cardiomyocytes from control, Drp-1 siRNA and Mfn2 siRNA group stained with potassium channel antibody (green fluorescence), sarcomeric actinin (red fluorescence) as a control labeling, B) and with PBFI dye (green) for intra cellular potassium. Dapi (blue) staining representing nuclei. Data represents mean $\pm \mathrm{SE}$ from 
$\mathrm{n}=5$ per group; * $\mathrm{p} \leq 0.05$ compared to control and Drp-1 siRNA; \#p $\leq 0.05$ compared control. 


\section{Mitochondrial membrane permeability}
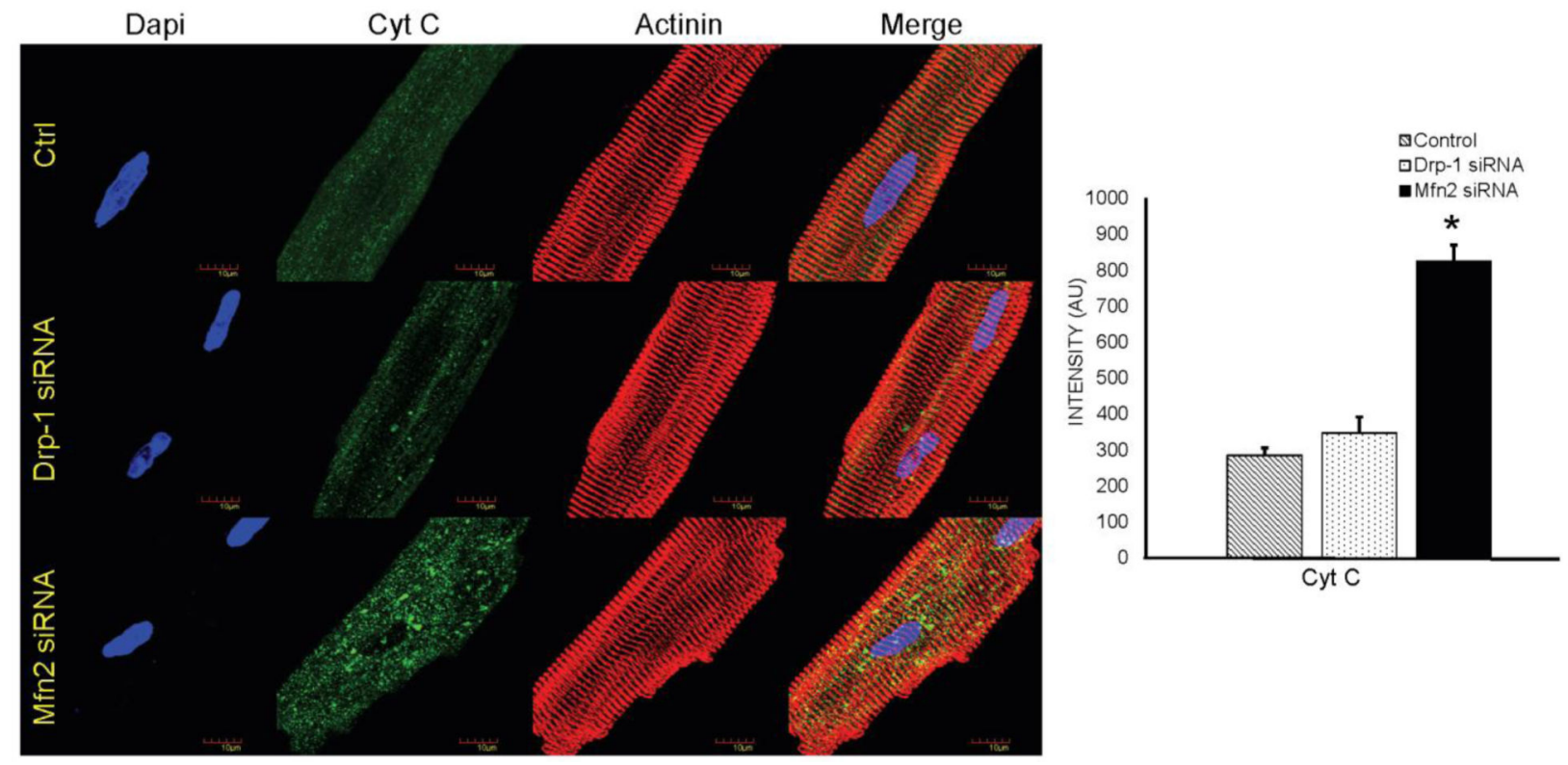

Figure 6.

Immunocytochemistry images of cardiomyocytes from control, Drp-1 siRNA and Mfn2 siRNA group stained with cytochrome $\mathrm{c}$ antibody (green fluorescence) for mitochondrial membrane permeability with control sarcomeric actinin (red fluorescence) antibody. Dapi (blue) staining representing nuclei. Data represents mean \pm SE from $n=5$ per group; *p $\leq$ 0.05 compared to control and Drp-1 siRNA. 
ICC images of Serca-2a
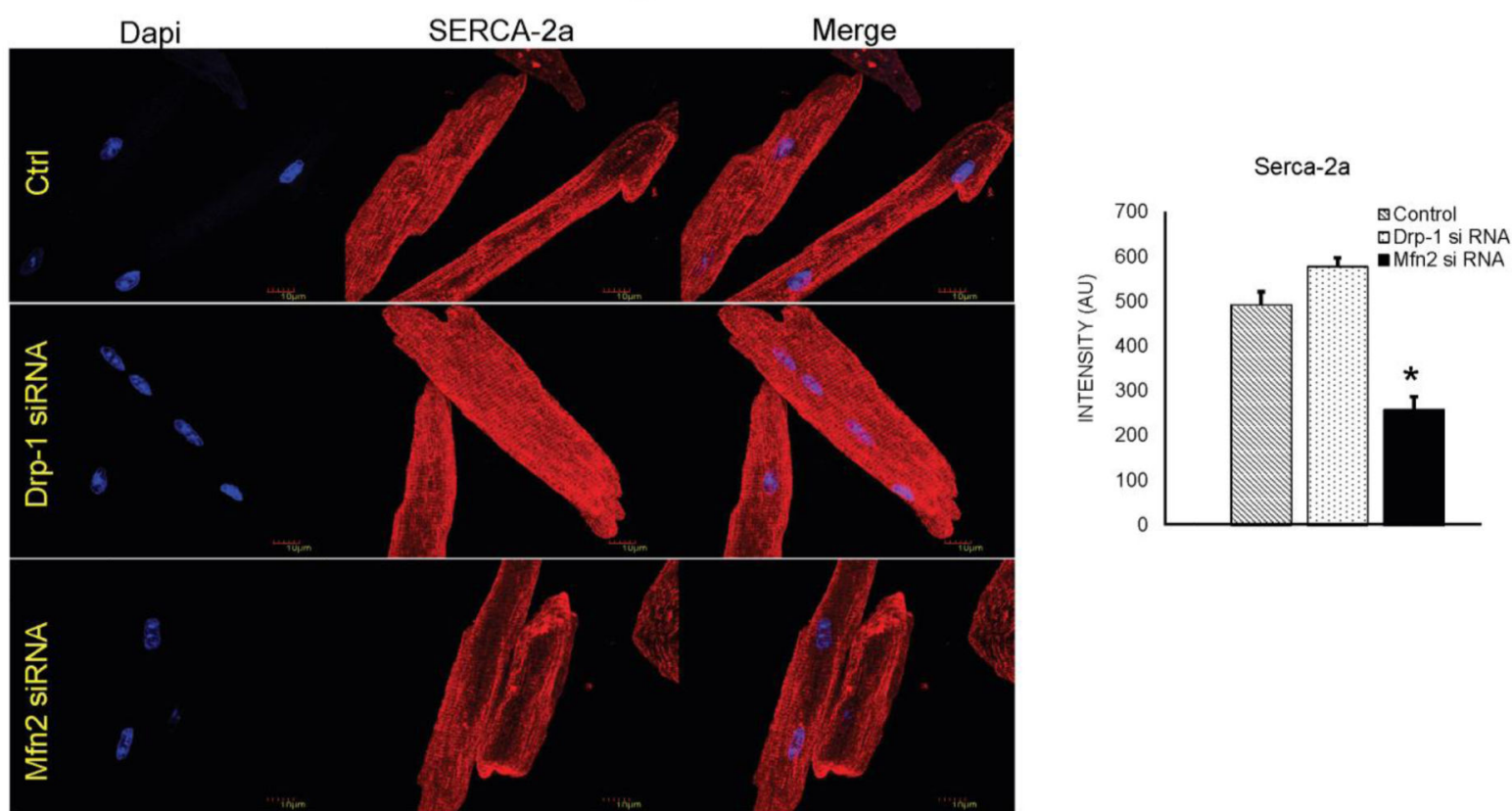

Figure 7.

Immunocytochemistry images of cardiomyocytes from control, Drp-1 siRNA and Mfn2 siRNA group stained with Serca-2a antibody (red fluorescence) and dapi staining blue represents nuclei. Data represents mean \pm SE from $n=5$ per group; ${ }^{*} p \leq 0.05$ compared to control and Drp-1 siRNA. 

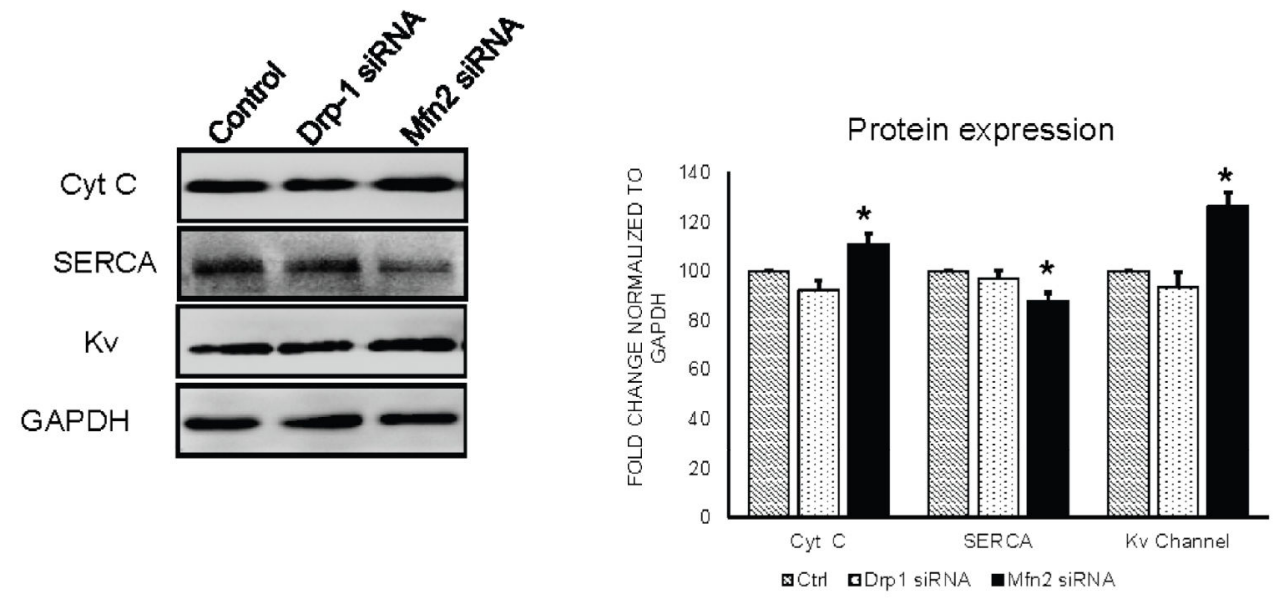

Figure 8.

Western blot analysis of cytochrome c, Serca and potassium channels expression in control, Drp-1 siRNA and Mfn2 siRNA group of myocytes. Fold change densitometry analysis of normalized protein expression with GAPDH in arbitrary units was depicted in bar diagram. Data represents mean $\pm \mathrm{SE}$ from $\mathrm{n}=3$ per group; * $\mathrm{p} \leq 0.05$ compared to control and Drp-1 siRNA. 

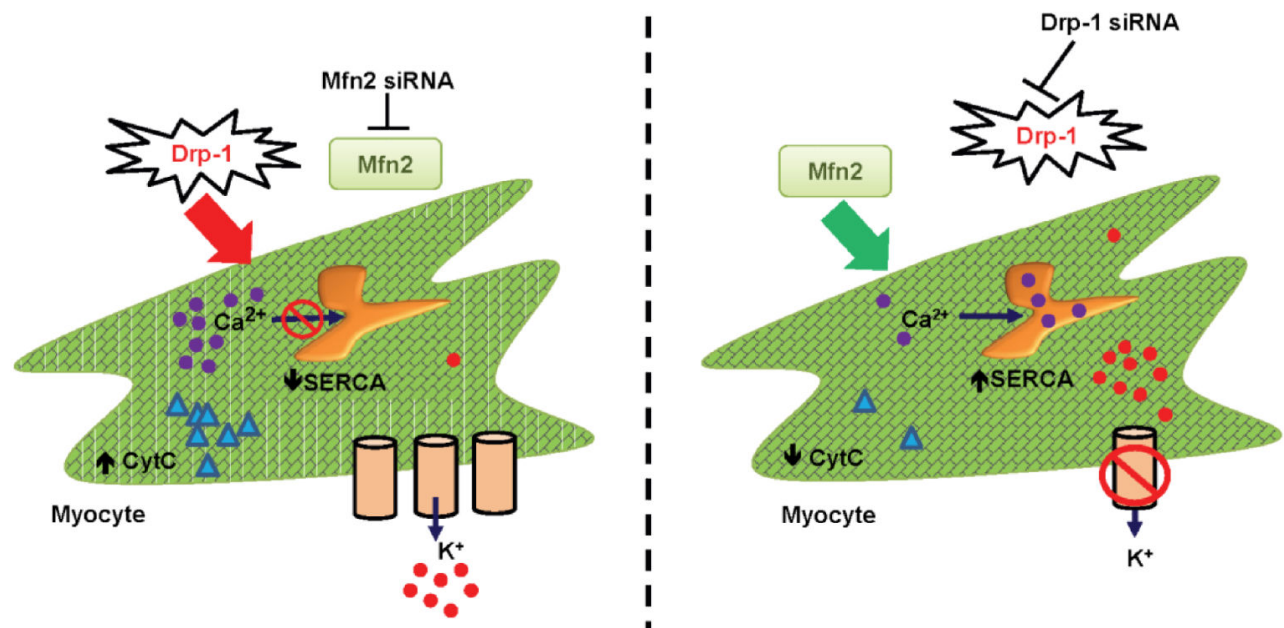

Figure 9.

Schematic hypothesis of mitochondrial fission and fusion regulating myocyte contractility. 